\title{
SOA-Based Supply Chain Performance Evaluation
}

\author{
Lin Zhisong \\ Ordnance Engineering College \\ Ordnance Engineering College, OEC \\ Shi Jiazhuang, China \\ 463716447@qq.com \\ Wang Jia \\ Ordnance Engineering College \\ Ordnance Engineering College, OEC \\ Shi Jiazhuang, China
}

\author{
Li Liangchun \\ Ordnance Engineering Institute \\ Shi Jiazhuang, China
}

\author{
Song Guifei \\ Ordnance Engineering Institute \\ Shi Jiazhuang, China
}

\begin{abstract}
Service-oriented architecture (SOA) is an application architecture. From the lack of research Supply Chain Management Performance Evaluation Methods exist at home and abroad, combined with supply chain performance management needs, article described Definition and characteristics of SOA. Than proposed a serviceoriented supply chain performance management evaluation system architecture.
\end{abstract}

Keywords- SOA; supply chain management; performance evaluation; system architecture

\section{INTRODUCTION}

According to supply chain management and operation mechanism of the basic characteristics of the target, the supply chain performance evaluation should be able to operate a proper response relationship between operational status and overall supply chain nodes up and down between enterprises, enterprises not only to evaluate the operational performance of the node, but also consider the impact of the node operating performance of its businesslevel node or the entire enterprise supply chain, and the traditional performance evaluation system is clearly unable to adapt to this model. Service-oriented architecture SOA is an application architecture in which all functions are defined as independent services can be invoked with a clear interface, you can call to order these services are defined to form business processes. Given the factors and methods of supply chain performance evaluation involves a very complex, integrated under evaluation information barriers and other issues, as well as the changing conditions of the market economy, performance evaluation requires a high degree of timeliness, comprehensiveness, accuracy and convenience, we introduce service-oriented the idea is established based on supply chain performance management evaluation system architecture of SOA, to solve the problem of supply chain performance evaluation has an important reference.

\section{ReSEARCh Status EVAluATION OF SUPPLy Chain PERFORMANCE}

Supply Chain Performance Evaluation (SCPM), supply chain around the target, the whole supply chain, each link (in particular, the relationship between the core business operations as well as various aspects of operating conditions among etc.) carried out before, during and after the analysis and evaluation (Neely et al., 1995). After the 1990 s, with the product-oriented market perspective to customer-oriented view of the gradual improvement of the market transformation, the market really began to bid farewell to the competition between enterprises and forms, replaced by competition between supply chains This confirms the competitive point of view of supply chain management guru Martin Christopher made in 1992 between the supply chain. Competition has become a key factor affecting the market competitiveness of enterprises cluster supply chain between the theory and methods of its evaluation has also been unprecedented attention.

\section{A. Performance Evaluation of Supply Chain}

Domestic and foreign scholars $^{[1-3]}$ for supply chain performance evaluation focused on selected systems to establish supply chain performance and supply chain performance evaluation, the evaluation system was created to focus on the balanced scorecard and supply chain operations based on Reference Model.

First, the performance evaluation of supply chain based on the Balanced Scorecard ${ }^{[4]}$ (BSC) is. In 1992 the famous American scholar Kaplan and Norton in the "Harvard Business Review" published a report entitled "The Balanced Scorecard: Drive performance evaluation system," the article, for the first time introduced the BSC performance evaluation in the field, followed by a series of published articles, and gradually formed a BSC model today. BSC model to overcome the deficiencies of traditional performance evaluation focus only on shortterm financial targets, from a financial point of view, four aspects of the customer perspective, internal process perspective, the perspective of learning and innovation in the enterprise strategic goals into specific objectives and 
performance goals, to achieve a comprehensive assessment of corporate performance purposes. BSC is not only an evaluation system and is a manifestation of management thinking through the financial indicators and non-financial indicators, internal and external environment, performance drivers (in the future, in advance) and the results (in the past, the lag) and Short-term indicators and long-term indicators combine to make the manager's attention away from short-term goals to achieve strategic goals into account.

Second, based on supply chain operations reference model (SCOR) supply chain performance evaluation ${ }^{[5]}$. Supply Chain Council developed and designed Supply Chain Operations Reference model (SCOR). Its basic idea comes from business process reengineering, benchmarking and best practices in three aspects. Model of supply chain management processes are generally defined performance benchmarks correspond to these processes, describes "best practices" (best practices) of the supply chain and choose four parts supply chain software product components. The level of detail according to the process definition is divided into three levels, each layer can be used to analyze the operation of the enterprise supply chain. Supply Chain Performance Evaluation System Based on Supply Chain Operations Reference model is developed to perform the corresponding characteristics and evaluation index based on the SCOR model describes the contents of each layer in order to achieve supply chain performance evaluation. As the greatest impact on the application of the most extensive reference model, SCOR supports the enterprise, external evaluation and improvement of business processes to make strategic business management possible.

In addition, the supply chain performance evaluation also using AHP, DEA (Date Envelopment Analysis) method, rough set and BP neural network, catastrophe progression method, fuzzy hierarchy comprehensive evaluation method and other methods.

\section{B. Existing supply chain performance evaluation problems}

Evaluation of the amount of information, data collection difficulties required. Existing supply chain performance evaluation index system involves two aspects, the vertical aspects of the process from the enterprise level to the management on the basis of the strategic level, the level of respect from the supply chain upstream (suppliers) to supply chain downstream (customer). With the advent of the era of economic globalization, supply chain lengths getting longer, the need to collect more and more information. Difficulty in practice using existing methods for evaluating the performance of the supply chain growing wider, often difficult to obtain complete data evaluation. In the case of incomplete data, quantitative analysis will produce bias, leading to differences in the actual status of the evaluation findings and significant.

Evaluation of information obtained interrelated, there are repeated. Among the collected evaluation of the supply chain, wherein the interrelated considerations, the information representative of the indicators has a great degree of duplication. Fundamental processes within the enterprise side indicators bearing enterprise information management strategy level indicators carry duplicate information. Carried downstream end of the supply chain information is carried by the upstream end of the supply chain information through the supply chain process variability after. Upstream end of the supply chain can not guarantee the information carried by the variation in the supply chain process entirely, in the case of duplication of data, quantitative analysis of deviations arising from evaluation findings and make the actual status of a significant difference.

Enterprise obvious characteristics, supply chain characteristics are not prominent. Supply chain performance evaluation theory is based on nearly a decade of enterprise performance evaluation theory evolved, with a strong enterprise features. Follows the four dimensions for performance evaluation of supply chain performance evaluation method based on the balanced scorecard, from a financial, customer, process and learning and growth in four areas of the supply chain for evaluation. Its essence is the corporate equivalent of some supply chain, and the supply chain as an extension of the enterprise. Here ignores the supply chain "chain" feature.

\section{SERVICE-ORIENTED ARCHITECTURE SUPPLY CHAIN PERFORMANCE EVALUATION}

Based on the current lack of supply chain performance management evaluation methods exist, this paper introduces a service-oriented thinking, by building an evaluator and evaluation for all types of objects supply chain performance evaluation platform to provide direction to solve or avoid these problems.

\section{A. SOA definition and characteristics ${ }^{[6-8]}$}

With the continuous development of network applications, in order to reduce heterogeneity, enhance interoperability and to meet the changing needs of users and other issues, Gartner presents a new software architecture in 1996 - a service-oriented architecture (Service -oriented Architecture, SOA). So far, SOA has not been a single, widely accepted definition, it is because there is an overlap between the business areas and SOA technologies involved. SOA is an architectural model, which can be loosely coupled, coarse-grained application components distributed deployment, and use a combination of the network according to demand. Service layer is the foundation of SOA can be applied directly invoked in order to effectively control the system artificially dependent software agents to interact with.

SOA is essentially a collection of services, interservice communication with each other, this communication may be simple data transfer, it could be two or more services coordinating some sort of activity. Between services requires some method to connect. The so-called service is precisely defined, improve packaging, which is independent of other services in the environment and state functions. Service-oriented architecture include service providers, service requesters and service agents who are respectively responsible for service publishing, service discovery and service binding three operations. Service operator who publish services to the service agent. Find a service agent through a service requester who needed services, and bind to these services, the service provider and service requester can interact. Building SOA services, and use standard methods to reuse and integration services is the core mission of SOA. SOA service 
configuration allows the functionality of the existing system for extraction and packaging, can also be constructed based on the new business needs. SOA basic structure shown in Figure 1.

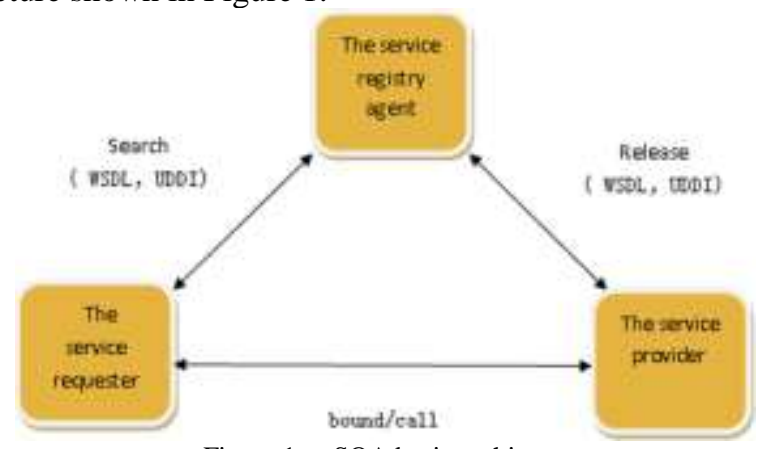

Figure 1. SOA basic architecture

Service-oriented thinking is the core of SOA, SOA is a services-based SOA-based systems integration program with the following features:

Coarse-grained: Based on the system through a set of coarse-grained services that can achieve different business processes and applications;

Loose coupling: coupling mainly to describe the dynamic relationship between providers and users invoke services between the provider can modify the service implementation process, without notice, and will not affect service users call to service ;

Encapsulation: SOA software module has certain functions packaged as a standard service interface for users to query service calls;

Reusability: SOA services provide different services available to users called repeatedly, thereby reducing software development and maintenance costs, improved efficiency and utilization of the software;

Cross-platform: SOA deployment can be integrated in different operating systems and network environments;

Language-independent: refers to different versions of the different language system call to the service can be achieved, so that it becomes possible to integrate between them;

Transparency: the use of SOA implementations and services will be separated from the service, the service does not have to be concerned about is the user service implementation details (including implementation services, is the location, transport protocol service), and the interface remains unchanged as long as the service, it will not affect the service call to the service user to achieve a completely transparent to the user;

Service interface standardization: standardized description of the service interface allows service users to become any platform and can accept a standardized interface.

\section{B. Supply Chain Performance Evaluation Based on SOA architecture}

SOA applications in supply chain performance management is an inevitable trend, which will help Chinese enterprises to effectively enhance the global competitiveness in the market. But not many domestic research is not deep enough, combined with SOA to solve practical problems. In this paper, supply chain performance evaluation system architecture based on SOA
${ }^{\text {[9-12] }}$ as shown in Figure 2. BM pointing benchmarking method, DEA refers to data envelopment analysis.

SOA-based supply chain performance evaluation system uses a hierarchical model, mainly by the business application layer, the service bus layer, and basic service layer:

Infrastructure services layer functions provide external evaluation services and information sharing services. Service evaluation function is to evaluate the various existing models in the form of service packages, ignoring the specific implementation details of each method, external standard service model, the service can be combined to create new services as needed; Information sharing services means from At all levels of suppliers, manufacturers, distributors, retailers, and ultimately consumers core businesses of various types of information and data evaluation and other services in the form of a text released to provide decision support for supply chain performance management.

Service Bus provides a service-oriented infrastructure, a key component, mainly to complete registration services, publishing and other aspects involved in providing service management, message transformation, message routing, protocol conversion, security management and configuration management. The layer technology involved is already a mature technology, such as Enterprise Service Bus (ESB).

User service application layer includes members at all levels of the supply chain (suppliers, manufacturers, distributors, retailers, the core business) and end consumers as well as other "chain" outside the component will become a network-based supply chain performance evaluation A system of the network node, the physical layer and the information layer having a plug and play function, various application services are open. The system architecture is more emphasis on the overall application performance evaluation system, in order to improve the competitiveness of the supply chain from the information provided technical support level.

\section{CONCLUSION}

SOA loosely coupled architecture provides a standardized, you can put various existing evaluation model package for the service, the service can also be provided to the new interface, thus bringing to evaluate supply chain agility and other benefits. In this paper, based on supply chain performance evaluation system architecture of SOA, integration of the whole supply chain resources in all aspects of information, can effectively solve the data collection is difficult to evaluate the information easy to duplicate, supply chain characteristics are not prominent, but not re-evaluation of multiple theories actually Applications and other issues, and to serve as the basis of its characteristics, enabling customers to evaluate more convenient, more timeliness. It is believed, SOA applications will have good prospects in the supply chain performance evaluation. 


\section{REFERENCES}

[1] Wu Fang, Zhang Tao, Gu Feng. Customer-oriented supply chain performance evaluation $[\mathrm{J}]$. Industrial Engineering and Management, 2012.

[2] Jin Hongliang, Rong Guifan. Literature review of supply chain management performance evaluation $[\mathrm{J}]$. Chinese township enterprises accounting.

[3] Kearney. Performance Measurement System in Supply Chain Management [J]. Harvard Business Review, 1993 (2):. 33-35.

[4] Wang Huanyi. On the Balanced Scorecard in Supply Chain Performance Management. Chinese high-tech enterprises, 2009.

[5] Song Hua. Logistics costs and supply chain performance management $[\mathrm{M}]$. Beijing: People's Posts and Telecommunications Press, 2007: 30-39.

[6] Bibositan. Service-oriented architecture (SOA) guidelines: Business Value, Planning and Enterprise Roadmap [M]. Beijing: People's Posts and Telecommunications Press, 2008: 3-16.
[7] Davis. The Definitive Guide to SOA: by BEA Aqua logic Service Bus to achieve [M]. Beijing: Electronic Industry Press, 2008: 6-10.

[8] Scott Campbell. Mastering Enterprise SOA with SAP Net Weaver and my SAP ERP $[\mathrm{M}]$. Changchun: Jilin Changbai Mountain Press, 2007: 23-32.

[9] Gu Xiaolin. Research on Supply chain management applications SOA performance evaluation method [J]. Productivity Research, 2009.

[10] Cheng Feimo. SOA-based integration of information resources of commercial banks. Computer Knowledge and Technology, 2009.2.

[11] Lin Qisheng. Research and Implementation of SOA-based enterprise-class e-commerce trading platform [D]. Engineering master's degree thesis, 2005.3.

[12] Huo Jia Zhen, Leixing Hui, Sui Minggang. Based on supplier performance evaluation system of supply chain [J]. Shanghai University (Natural Science), 2002 (2) 2: 177-188.

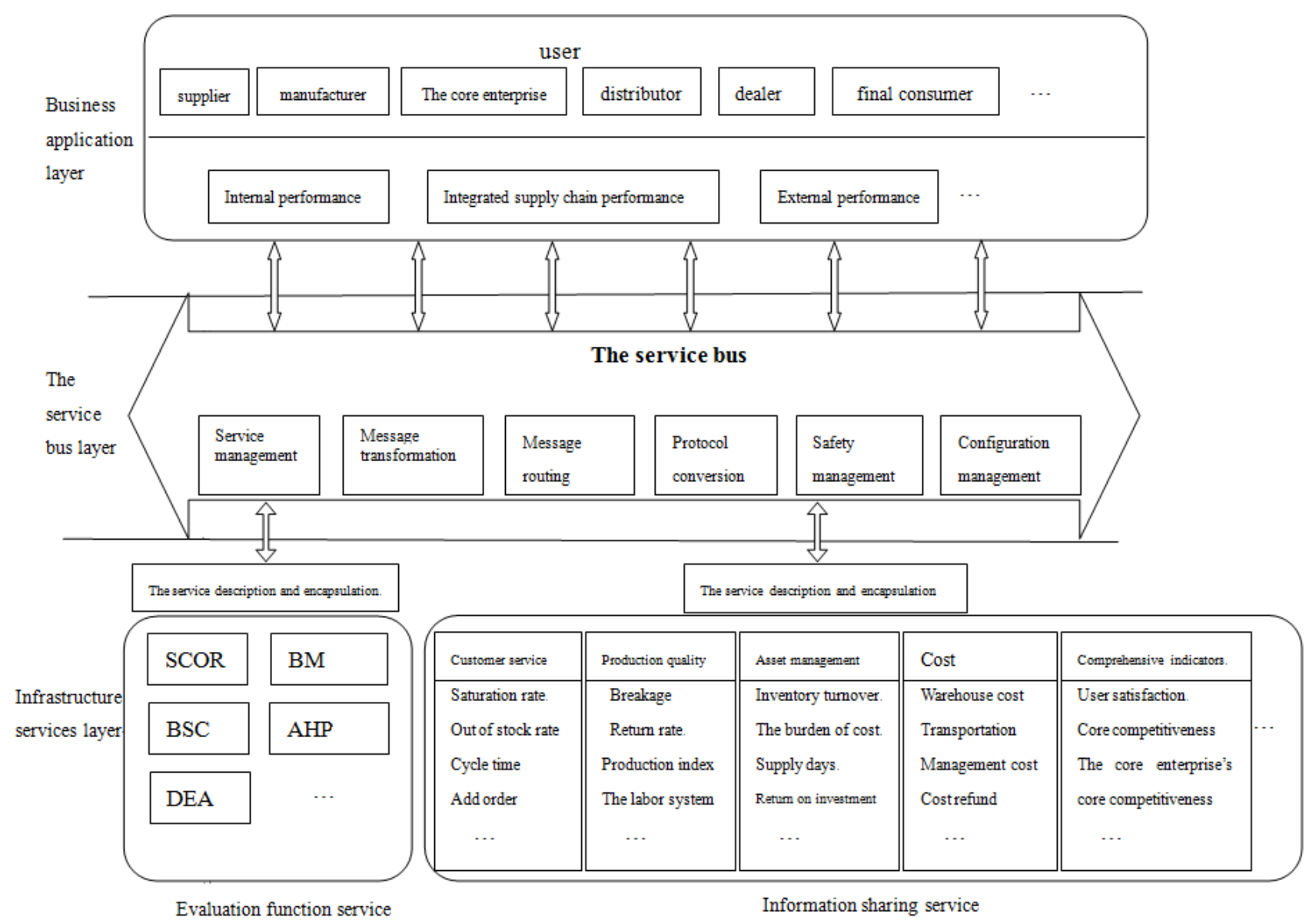

Figure 2. Supply Chain Performance Evaluation Based on SOA architecture 